\title{
Germanica
}

$11 \mid 1992$

Le Conte dans les littératures germaniques du XXe siècle

\section{Une lecture du monde : Die Abwesenheit, un conte de Peter Handke}

Eine Lektüre der Welt: Die Abwesenheit, ein Märchen von Peter Handke

\section{Laurent Margantin}

\section{(2) OpenEdition \\ Journals}

Édition électronique

URL : http://journals.openedition.org/germanica/1352

DOI : 10.4000/germanica.1352

ISSN : $2107-0784$

Éditeur

Université de Lille

Édition imprimée

Date de publication : 1 janvier 1992

ISSN : 0984-2632

\section{Référence électronique}

Laurent Margantin, «Une lecture du monde : Die Abwesenheit, un conte de Peter Handke », Germanica [En ligne], 11 | 1992, mis en ligne le 07 février 2014, consulté le 06 octobre 2020. URL : http:// journals.openedition.org/germanica/1352 ; DOI : https://doi.org/10.4000/germanica.1352

Ce document a été généré automatiquement le 6 octobre 2020.

(c) Tous droits réservés 


\section{Une lecture du monde : Die Abwesenheit, un conte de Peter Handke}

Eine Lektüre der Welt: Die Abwesenheit, ein Märchen von Peter Handke

Laurent Margantin

1 «Diesen Abend verspreche ich Ihnen ein Märchen, durch das Sie an nichts und an alles erinnert werden sollen». En exergue à Die Lehre der Sainte Victoire, cette citation de Goethe a son importance. Elle suggère que déjà au moment de la rédaction de ce livre, en 1980, l'écriture du conte attire Peter Handke. Oui, il y a encore un monde merveilleux à explorer, nous dit l'écrivain, monde merveilleux que n'explore pas le conte traditionnel, mais qu'exprime la peinture, et plus particulièrement celle de Cézanne. Et plus loin dans le même livre, on pourra lire : «[...] geltend waren gerade die Normalsachen, die aber der Maler in den Schein des Besonderen gestellt hatte - und die ich jetzt die "magischen" nennen kann». Die Abwesenheit, sept ans plus tard, raconte ce passage de l'homme à une réalité supérieure, à ce qu'Handke appelle le «Réel des réels ", qui n'est nullement rupture avec le paysage, mais au contraire union avec lui, au moyen de l'écriture-dessin. Le conte peut permettre de dire ce passage, car il est toujours récit d'un voyage "de l'autre côté », dans l'invisible. Mais la nature de celui-ci est transformée : il s'agit d'accéder à une « sensation vraie » du monde, et non plus à un univers irréel. Comme Handke lui-même dans Die Lehre der Sainte Victoire, quatre personnages, le vieil homme, le joueur, le soldat et la femme - tout le long du récit ils resteront anonymes - partent à la recherche d'un «Hochland», haut-pays où l'esprit s'éveille à la matière. Pour quitter la ville, ils prennent un train spécial qui les mène non loin d'une forêt. Ils traversent cette forêt, découvrent une clairière idyllique, puis la quittent pour rejoindre une rivière. Après l'avoir longée un moment, ils font l'ascension d'une montagne en haut de laquelle ils s'initient à ce qu'on peut appeler une « lecture du monde ». Voilà, vite tracé, le parcours. Mais retournons d'abord au manque initial, condition du départ, et observons en détail le cheminement initiatique que décrit le conte de Handke. 
2 Avant qu'ait lieu le départ en train, chacun des personnages souffre en effet d'un manque. Celui-ci est évoqué au début du récit, soit par le personnage concerné, soit par l'un de ses proches. La femme est accusée par son ancien compagnon de ne pouvoir s'ouvrir aux autres et à tout ce qui est extérieur, étranger à elle-même. Quantité de photos, ne représentant qu'elle, décorent les murs de son appartement, et elle passe beaucoup de temps devant son miroir. À cause de son égoïsme, elle ne peut être aimée, et devra donc quitter sa chambre et son miroir pour trouver l'amour, c'est-à-dire s'ouvrir à la beauté de l'Autre (paysage ou être). Comme la femme, le soldat est absent du monde qui l'entoure, il n'a pas de réels contacts avec ses semblables, même avec ses parents. Il est l'inconnu, l'invisible, celui qu'on ne remarque pas, et sa mère lui dit : «Du bist da, und zugleich nicht da. [...] Dein Abwesendsein vertreibt uns aus deiner Nähe». Lui aussi devra partir, rencontrer d'autres hommes, découvrir une convivialité inédite auprès de ses trois futurs compagnons. Le troisième personnage qui devra rompre avec un quotidien étouffant est le joueur. Travaillant dans une salle de jeu (il distribue des cartes et lance des dés sur une table, sans que le lecteur puisse deviner à aucun moment de quel jeu il s'agit), il en est le personnage central, et pourtant, personne ne semble faire attention à lui ; sa présence est en réalité une absence pour les autres joueurs qui lui font face. Bien que riche, il désire une vie plus aventureuse : «ANFANGEN. Ja, ich sage das jetzt zum ersten Mal... Ich werde ein neues Leben anfangen».

Comme on le voit, les problèmes de ces trois personnages paraissent banals, et ressemblent aux nôtres. L'espace premier du conte est social, espace dans lequel chacun étouffe de ne pouvoir établir de communication. Il ne s'agit pas pour le joueur, le soldat et la femme de partir à la recherche d'un royaume imaginaire. Leurs désirs se résument à celui-ci, essentiel : toucher enfin ce qui est le plus proche, ce qui devrait être le plus facile à atteindre. La base du conte de Handke est véritablement moderne : les personnages ne cherchent aucune révélation divine, aucune morale, mais un bienêtre. Tout ce que voudra montrer le conte, c'est que ce bien-être n'est pas un nouvel " avoir ", mais un nouveau regard, ce dont, au départ, ne sont pas toujours conscients les personnages.

Pour guider le joueur, le soldat et la femme vers le haut-pays, il y a le vieillard, pensionnaire, au début du conte, d'une maison de retraite. Celui-ci est le personnage le plus important du conte, celui qui livrera un secret, ou une série de secrets, à ses trois " disciples ». Il vit dans un monde parallèle, monde à côté du monde moderne, monde des arbres et des chemins, des lacs et de l'océan, et fait souvent penser au personnage légendaire de Merlin l'Enchanteur. Il a un pouvoir : celui d'entrer en contact avec les choses, et de les exprimer. S'il écrit, c'est à la recherche de signes exprimant un sentiment complexe du réel, dans une tension incessante vers un autre langage. Il ne peut y avoir pour lui de rapport profond avec ce qui l'entoure que par le biais d'une écriture mystérieuse qu'il trace sur un carnet de toile, attentif aussi au dessin de celleci : «Die Seiten sind, in senkrechten Reihen, zum Teil bedeckt mit Zeichen, die an Bilderschriften erinnern, ohne dabei bestimmbar zu sein». Plusieurs fois il sera appelé "der Entzifferer», «le décrypteur ", celui qui, déchiffrant le monde pour en trouver les formes, en trouvera du même coup la langue, langue profondément inscrite dans les choses. Ce thème de l'écriture est essentiel, puisque les trois autres personnages du conte sont eux aussi à la recherche d'un verbe. Dans la première scène où elle intervient, la femme rédige une lettre, mais son écriture reste illisible : «Trotz der Blockbuchstaben bleibt ihre Schrift unleserlich: die wenigen sich einer Form 
nähernden Zeichen haben den Schwung chinesischer Kalligraphien». Le soldat est continuellement plongé dans un livre, et il y cherche une vision propre. Le joueur lui aussi voudrait trouver une parole: «Buchstabieren. Schriftlich geben. Ja: Damit ich meine, was ich sage, und damit es Gehör findet und gilt, werde ich es buchstabieren und mir schriftlich geben. Gibt es auch das Besungene nicht, so doch die Stimme des Sängers». Il est important de remarquer au passage que l'écriture est souvent comparée dans Die Abwesenheit au chant; la parole doit s'élever dans l'air et enchanter le lecteurauditeur. Cette conception d'une écriture tendant vers le chant nous amène à penser que le conte lui-même retrouve sa fonction archaïque: celle d'être raconté à voix haute, rythmé, afin de charmer un auditoire. Il n'est plus un texte que l'on doit lire pour soi seul, à l'écart, mais redevient la parole d'une communauté, aussi disloquée et malade soit-elle.

Ce désir d'écriture est envisagé par Handke comme une puissance, puissance magnifiant celui qui la ressent au plus profond de lui-même. Ainsi le vieil homme, parce qu'il tente à chaque instant de vivre dans la jouissance du réel que peut lui permettre l'écriture, nous apparait vite comme un personnage légendaire. Au début du récit nous le voyons dans la chambre de l'hospice tenant une canne "comme un spectre ", et Handke ajoute: "Der Bewohner, der am Fenster steht, ist kein Heiminsasse, sondern der Herr dieses Raums». S'il est souvent représenté comme un roi, il est aussi comparé à un savant, et parait de toute façon issu d'un monde ancien, où le sacré jouait encore un rôle. Nous retrouvons aussi la figure du roi dans le portrait du joueur. Il trône lui aussi, mais cette fois dans une salle de jeu, et non plus dans la solitude d'une chambre d'hospice. Quant à la femme, elle est comparée par son amant à une sorcière, à Circé qui transforme les compagnons d'Ulysse en pourceaux. En face de son miroir, elle ressemble à la reine démoniaque de Sneewittchen, un conte de Grimm, qui demande au sien :

Spieglein, Spieglein an der Wand,

Wer ist die Schönste im ganzen Land?

Mais surtout, tous les quatre détiennent des pouvoirs magiques. Le soldat est transparent, invisible; le vieillard fait se métamorphoser le paysage sous les yeux du lecteur ; la femme porte une valise pesante comme s'il s'agissait d'une plume. Par leur caractère légendaire les faisant ressembler à des héros de contes traditionnels qui nous sont souvent familiers, ils rompent avec leur "identité ", celle qu'évoquent leurs dénominations (en rapport avec leur fonction sociale, leur âge ou leur sexe). Ils se débarrassent d'une ancienne apparence qui n'était que déguisement, tromperie, jeu social, et font apparaître leurs véritables identités, identités symboliques. Celles-ci sont nombreuses et s'opposent à l'identité sociale étouffante du départ; le vieil homme se révèle tout à la fois géographe, magicien, chaman (celui qui fait migrer les âmes), et le limiter à la figure du roi serait restreindre ses pouvoirs.

6 Nous pouvons même voir dans ces personnages plus que des figures légendaires, figures encore trop humaines, et lire à travers eux, à travers leurs actes et quelquefois leur caractère, la symbolique alchimique du conte. Les quatre personnages, ou héros de Die Abwesehheit, représentent en effet les quatre éléments indispensables au Grand CEuvre: le joueur symbolise la Terre; le vieil homme, l'Eau; le soldat, l'Air; et la femme, le Feu. De plus, à chaque élément correspond un stade du voyage initiatique, ou une portion du chemin. C'est le joueur qui inaugure le voyage alchimique: nous le voyons dès le début en accord avec la terre, fuyant les rues asphaltées de la ville : «Der Spieler liegt auf der Erde, im Steppengras, mit dem Gesicht nach unten, angestrahlt von 
einer Friihlings sonne». Certes, cela seul ne suffit pas pour associer le joueur avec l'élément terrestre. L'association devient totalement justifiée lorsqu'une fois descendus du train, les quatre compagnons s'avancent dans la forêt.

La seconde phase est celle de l'eau, lors de laquelle le vieil homme à son tour guidera les autres le long de la rivière jusqu'aux premiers escarpements de la montagne. Sa souplesse de jeune homme évoque aussi la fluidité de l'eau, et il se baigne souvent, se retrouvant alors, comme on dit, «dans son élément ». À la fin du récit, lorsque le vieil homme aura disparu, les autres auront soif ; quantité d'autres détails de cette espèce rappellent sans arrêt cette identité symbolique de l'«Entzifferer». Ajoutons qu'à l'eau des alchimistes est toujours liée l'imagination, et que c'est là une qualité du vieillard, lui qui sait voir au-delà, plus loin que le monde moderne le haut-pays oublié.

$8 \mathrm{Au}$ sommet du «Hochland», le soldat est enthousiasmé par le paysage, paysage désertique, parcouru sans cesse par le vent, "littéralement enthousiasmé de ne plus savoir où il (est) comme quand on s'éveille loin de chez soi, quelque part dans l'incertain et qu'on est débarrassé de son nom, mais en revanche définitivement prêt pour le matin, la lumière, la sortie à l'air libre, les gouttes de pluie dans la poussière, les yeux du premier venu, les mots du vieux livre» (traduction de Georges-Arthur Goldschmidt). Ces quelques lignes d'une extraordinaire fraîcheur illustrent bien l'exaltation du soldat lors de ses retrouvailles avec son élément, l'air, comme lui invisible, insaisissable («Du bist Luft» lui dit sa mère dès les premières pages).

Quant à la femme, si nous pouvons reconnaître en elle le Feu, c'est parce que le feu est le dernier élément intervenant dans l'œuvre alchimique, chargé de brûler les éléments impurs qui agissent dans la fabrication de l'or, et que la femme, dans la dernière scène réunissant les quatre personnages, allume un feu (dans une grotte, lieu convenant bien à une sorcière), avant d'attaquer le vieillard, dénonçant ses pouvoirs qui ne sont pour elle que poudre-aux-yeux. Après cette violente diatribe, le vieil homme se promène dans un paysage déserté, comme brûlé, et disparaît. Nous reviendrons sur cette disparition, centre du conte. Contentons-nous d'ajouter, pour finir de justifier notre association femme-Feu, que l'héroïne plonge une fois ses pieds dans les braises d'un feu de camp, le plus naturellement du monde...

Comme nous l'avons vu, cette symbolique «élémentaire» structure le temps de l'initiation; elle va même jusqu'à diviser celle-ci en saisons. La phase terrestre se déroule au printemps. Puis, lorsque les personnages commencent à longer la rivière, c'est l'été : «Am Morgen ist es dann Sommer geworden». Suit, en haut de la montagne, le commencement de l'automne, saison, dans la symbolique alchimique, de l'accomplissement, de l'« CEuvre-au-rouge». Seulement, si Handke emprunte à la tradition hermétique quelques-uns de ses plus beaux symboles, ce n'est pas pour conclure sur un quelconque accomplissement; nous verrons qu'au sommet du hautpays se produit ce que nous pourrions presque appeler un drame. Aucun des quatre personnages n'atteint, comme pourrait le laisser supposer le cheminement initiatique précédemment exposé, la sagesse... C'est ce que nous dit, parmi beaucoup d'autres choses, le conte de Handke : il n'y a pas à rêver, si l'on renoue des contacts profonds avec le monde, d'un accès à une vérité, à ce que les philosophes anciens et parfois modernes appellent l'Être, - il ne peut y avoir qu'une tension bénéfique, enrichissante et avant tout libératrice vers une sagesse de toute façon, et heureusement! inaccessible. Et bien entendu cette tension peut être interrompue par le doute et le 
désespoir, comme dans ce passage qui suit ce « simulacre d'accomplissement », passage où le vieil homme a disparu, obligeant les trois autres à tout recommencer.

Par ailleurs, ce cheminement initiatique n'est pas si simple à réaliser. À chaque instant il faut faire effort, et peu à peu, difficilement, se dépouiller de l'ancienne identité sociale. Partir pour le "Réel des réels", cela veut dire, une fois parti, trouver son chemin...

12 Le départ lui-même est difficile. Il s'agit en effet d'un premier passage, ou d'une première épreuve. Le soldat quitte son poste et fuit en courant par un tunnel, transition obligée pour se retrouver " de l'autre côté », où, écrit Handke, « les haies au bord de la route ont des feuilles persistantes où étincellent des coupelles de soleil méridional ", espace déjà plus clair, riche de mille détails faisant oublier les ordres militaires, tout ce monde au garde-à-vous que laisse derrière lui le jeune homme. Le joueur sort de la ville, énorme salle de jeu, et dit une incantation (qui consiste littéralement à « opérer un charme ») au milieu d'un cercle magique. La femme, elle, doit trouver une issue à la ville devenue tout à coup labyrinthique.

13 Ces fuites, ces errances leur permettent d'accéder au train, condition sine qua non de leur accès au « Hochland».

14 Après ce passage décisif, le conte fourmille de seuils, de portes plus ou moins secrètes que franchissent un à un les personnages. On passe aussi souvent sous une arche, lorsque le train part par exemple :

Die Ausfahrt geschieht unter zwei Bäumen - der eine an den Schienen, der andre an der begleitenden Strasse-, deren Ast werk ineinander gewachsen ist und einen Bogen bildet.

15 Cette figure de l'arche réapparaît souvent dans le conte, symbole du commencement, de l'ouverture sur un monde nouveau. Nous avons également vu auparavant qu'à l'entrée de la forêt le mur de terre représente une frontière. De même, avant l'arrivée au haut-pays un «Grenzbaum» fait office de borne délimitant un territoire. Tout de suite après, un cimetière doit être traversé, et des signes troublants surgissent, comme ce «anwesend» gravé sur les tombes qui concerne autant les morts que les vivants enfin présents, morts eux aussi au monde ancien qu'ils habitaient. Comme dans de nombreux contes archaïques les âmes doivent opérer ce qu'on pourrait appeler une "transmigration", dans le sens où les personnages doivent bel et bien "changer de corps ", passer d'un corps insensible ou bien même opposé à ce qui l'entoure à un corps continuellement en liaison avec un monde débarrassé de tout ce qui peut aliéner l'homme. Bien sûr, rien de «mystique » dans ce « changement de corps ", il ne s'agit là que d'une image pour exprimer cette rupture interne que tentent les héros de Handke avec toute une partie de leur existence, rupture qui permet, ou doit permettre une métamorphose quasi totale de l'individu; ce moment est le pivot du passage de l'absence à la présence qui commande tout le livre de l'écrivain autrichien.

Il faudrait aussi parler du symbolisme de l'arbre aux branches qu'Handke compare à des serpents. C'est l'arbre du monde, le «Märchenbaum» que taille le vieil homme avant son départ de l'hospice, et qui est coupé en morceaux à la fin du récit. Il exprime toute l'ambiguïté du conte : est-il mort, cet arbre, ou ses racines sont-elles encore vivantes?

17 Ce foisonnement des lieux révélateurs nous transmet quelque chose d'essentiel : tout est magique, chaque chose nous ouvre les portes du « royaume du monde». Ce qui importe avant tout, c'est le mouvement, c'est l'aller libre qui nous débarrassent d'un regard impur, regard de l'habitude, de l'indifférence, et nous dévoilent la beauté du 
dehors. C'est ce qu'explique le vieil homme à la femme lorsque celle-ci veut rester dans la clairière, véritable jardin d'Eden :

An Ort und Stelle, gleichwo, hat die Erfüllung keinen Bestand. Sie wird entzaubert, im Handumdrehen, und mit ihr der Ort. Es ist nicht hier, wir sind nicht da. Auf. Weg von hier. Weiter. Hinaus. Es ist Zeit.

Certes il faut sortir de soi et de la ville, mais pour, ayant l'espace libre autour de soi, changer de regard. Il importe de savoir "visionner ", comme disait le peintre Dubuffet, c'est-à-dire de savoir lire les formes, les couleurs d'un paysage, comme on lit en profondeur un texte, en en découvrant toute la vie organique. Cela implique un exercice continuel, d'être toujours "sur le motif », donc de changer de lieu, de relief afin de ne pas, à nouveau, s'endormir. Et pourtant, quoiqu'il faille selon le vieil homme découvrir toujours de nouveaux lieux, celui-ci insiste sur le fait qu'il n'est pas nécessaire d'aller à la recherche de sites exotiques, que des lieux " neutres » suffisent. Les lieux de l'aventure sont proches, ils sont à quelques kilomètres de la ville, et deviennent lointains par la profondeur de la vision. Peter Handke nous fait ainsi découvrir un monde à la fois banal et merveilleux, tout proche et extrêmement éloigné, petit et vaste, un monde de contraires, de contrastes que les personnages et le lecteur avec eux doivent dépasser s'ils veulent atteindre une clairvoyance. En effet, Die Abwesenheit est riche en éléments contraires s'associant « comme par magie ». Je pense aux deux rivières vues depuis le train : «Dem von dem Zug überquerten Fluss, kaum ein Rinnsal zwischen den Schotterbänken, folgt ein zweiter, sich wälzend mit trüben Hochwasser, der vielleicht nur der nächste Mäander des ersten ist». Par ces visions courtes mais répétées le conte ouvre la conscience du lecteur à un monde paradoxal, complexe, insaisissable, réel. Le conte n'est plus alors l'histoire de la découverte de royaumes imaginaires, puisque la réalité elle-même provoque la sensation de l'irréel. C'est ainsi qu'à l'intérieur d'un même paysage on peut découvrir un autre paysage, comme l'« essence » du premier : observant un horizon d'arbres, le vieil homme voit apparaître des mâts, un atoll, tout un univers marin. Celui-ci reparaîtra continuellement par la suite, quelquefois simplement par touches, par exemple lorsque le train s'apprêtera à partir : «Ein Signal ertönt, kein Pfiff sondern ein Tuten wie von einem Ozeandampfer [...]». Plus loin, au commencement de l'ascension de la montagne, une barque est au bord du chemin, «l'air d'avoir été déposée là, sur la pente, en un temps préhistorique, lorsque la mer arrivait jusque là ». Aller vers le haut-pays, c'est aussi revenir à ce temps où l'océan recouvrait presqu'entièrement la Terre, c'est à la fois marcher vers un sommet et naviguer vers une île. L'espace devient multiplicité d'espaces, en un seul lieu surgit une variété de reliefs et de climats: «[...] das fremde Land kannso-wohl der hohe Norden sein, als auch der Tiefe Süden, als auch eine Gegend im Inland, neuartig durch das Licht eines besonderen Augenblicks». Il suffit, pour qu'apparaisse l'autre espace, que les formes de celui-ci se dessinent. De même pour les objets, ou les éléments de cet espace. Nous voyons le train passer plusieurs fois avant qu'il ne passe "réellement » : les lignes du parquet se changent en rails, ou bien les mots tracés sur la page en wagons... S'ouvrir au monde, c'est donc aussi laisser libre cours à l'imagination, difficilement séparable de la sensation des choses.

Cette ouverture à une diversité d'espaces entraîne du même coup une ouverture à une autre temporalité. Découvrant combien le réel est riche en paysages, les personnages ont l'impression que chacune des journées qu'ils vivent en contient plusieurs, ce qu'exprime le vieil homme dans la grotte : 
Wie viele Tage sind mir vergangen an diesem einzigen Tag! Einen Tag für sich schon hat es gedauert, euch beim Spiel zuzuschauen; einen zweiten, flussab zu gehen; einen dritten, hinauf ins Hochland zu steigen: einen vierten, sich davor erst einmal zu orientieren; eine ganze Woche habe ich dann gebraucht, die Wegemarkierungen zu entziffern, euch durch Regen und Wind zu meiner Tropf Sterngrotte zu führen.

Entré dans cet espace où tout est révélation, c'est-à-dire sensation, l'instant se dilate et vaut plusieurs heures, une seule journée une saison.

En racontant ce passage à un autre espace et à un autre temps, Die Abwesenheit dénonce la pauvreté d'un rapport au réel moderne. Symboles de ce rapport, l'autoroute et la télévision, présentes toutes les deux au début du conte. Elles sont les symboles du monde de l'absence, de ce monde où, pour interpréter le mot allemand, manque l'essence, manque l'être («ab-wesend»). Les téléspectateurs sont représentés justement comme les grands absents: «Auch die Fernseherlichter springen über die leeren Wände und wechseln die Farben wie für sich allein». Quant à l'autoroute, elle est cette ligne droite qui coupe l'homme du dehors, car obligeant le conducteur à se concentrer sur les dangers de la route elle lui interdit toute vision approfondie du paysage. Le téléspectateur et l'automobiliste ne font tous deux que s'engouffrer dans un monde d'images qu'ils ne créent pas et dont ils sont à chaque instant prisonniers.

Totalement opposé à ce monde de l'absence, au temps monotone et à l'espace citadin aliénant (labyrinthique), il y a le monde de la présence, ouvert, naturel, riche en détails enchanteurs. Celui-ci, cependant, ne peut être atteint qu'après un long effort de décryptage. Il ne suffit pas en effet de partir et de rompre avec le monde de l'absence pour accéder au haut-pays, il faut encore rompre avec une manière d'être et en élaborer une autre. Le vieil homme ne cesse pas tout au long du voyage de tenter de débarrasser ses compagnons de tout ce qui encombre leur esprit et les empêche, même loin de la ville, d'être ouvert au paysage. Il est le "trouble-fête", interrompant le joueur et le soldat quand ceux-ci oublient ce qui les entoure pour jouer avec passion aux cartes; dans un mouvement de colère ils jettent les cartes dans la rivière, bouts de carton qui ne sont que les traces d'un monde qui doit être oublié, et avec lui tous ses jeux, toutes ses règles. Il prend aussi à la femme son miroir, son seul refuge quand elle a peur de faire face au réel. Quoiqu'il en soit, ce geste du vieil homme, nous dit Handke, reste insuffisant : «Frau und Spieler Hessen es geschehen, als hätten sie für diese Dinge ohnehin die Doubletten bei sich». Ce n'est pas une mince affaire que de rendre à la nature des hommes dénaturés, attachés à une multitude de "suppléments" (on sent souvent en effet Handke fortement influencé par Rousseau...). D'où la nécessité du langage pour ces hommes dépendants de leurs objets, sorte de clé leur dévoilant la beauté du monde autour d'eux. «Der Schriftakt war das, was uns gefehlt hatte » dit ce "nous" intriguant du récit, "nous" de plus en plus présent, tout en demeurant inconnu, anonyme, et qui n'est autre peut-être que le «je » de l'écrivain, mais «je » fondu au groupe des personnages comme à d'autres images de lui-même, car ce «je ", dans l'acte d'écrire, vit la même aventure que les quatre acteurs de son livre. L'écriture elle aussi fait passer de la possession illusoire de quelques objets à une relation profonde avec le réel. Mais chez Handke, cette écriture équivaut à une lecture. L'«Entzifferer» doit pouvoir lire les formes du paysage, et les retranscrire; ce sont là deux temps d'un même acte. Le langage est dans les choses, inscrit en elles, il est les 
choses. Le paysage du haut-pays, comme le laisse voir ce passage - début de l'épisode sur le «Hochland» - est texte lisible :

[...] es scheint da, so weit das Auge reicht, keine versteckten Winkel zu geben, weder Taleinschnitte noch unsichtbare Hügelrückseiten. Die Gegenstände zeichnen sich dadurch klar und unverstellt ab, ein jeder vermittels der Grossform abgerückt und abgehoben vom andern zu einer selbständigen Kleinform und derart mit den Nebengegenständen verknüpft $\mathrm{zu}$ einer Vielfalt zusammengehöriger zierlicher Gestalt, die in dem Oval ein reges Legen, sogar eine frenetische Erwartung vortäuschen, so als sei da in Begeisterung eine festfrohe Menschheit versammelt.

Les choses et les mots tout à coup concordent; reste alors au vieil homme à faire la moisson (Ernte), et il s'y met après avoir convaincu les autres qu'un langage est vivant dans le réel qui les entoure, en faisant surgir d'une dalle, "comme un prestidigitateur ", une « image ", c'est-à-dire un cadran solaire sur lequel trois lettres sont lisibles: "dim», lettres que le vieil homme interprète ainsi: «Deo invicto Mithrae», ou " Au Dieu Soleil invaincu ». C'est en effet la lumière du soleil qui permet la moisson. Ce passage de la moisson est superbe, et rappelle ces tableaux de Van Gogh où un paysan, au milieu des champs bouleversés par le mistral, dans la violente lumière du Midi, fauche les blés. Celui qui lors de cette scène fauche avec véhémence, tandis que les autres s'initient à cette lecture toute nouvelle pour eux en essayant ici et là de glaner quelques signes le nez pointé vers le sol, le vieil homme, se change vite en véritable magicien, faisant apparaître une profusion de choses, plantes, oiseaux, nuages, et en même temps signes d'une écriture inédite. C'est de cette lecture-écriture qu'avait rêvé Handke devant les tableaux de Cézanne, extraordinaires rencontres, selon l'écrivain, de l'objet, de l'image, de l'écriture et du trait. Ici cette rencontre se réalise sur le papier, dans le dessin des mots ; car le geste de l'écrivain compte également, et le vieil homme trace d'un trait des mots dont la beauté est aussi d'être des images, l'écriture devenant quasi idéographique. Ainsi le peintre peut être égalé au moyen d'autres outils, et presque d'un autre "matériau ». La méditation de la peinture a pour conséquence nouvelle non pas la naissance d'une autre peinture mais d'une autre écriture, d'une autre pensée de l'écriture. Remarquons toutefois que Handke ne fait ici que poursuivre une recherche sans doute très ancienne, assez importante ce siècle-ci, qui consiste pour les écrivains à se tourner vers la peinture (autant traditionnelle qu'archaïque ou moderne) pour découvrir de nouvelles voies en littérature; je pense notamment à Francis Ponge, dont l'écrivain autrichien a traduit le Carnet du bois de pins, et qui chercha lui aussi dans l'écriture une union avec le monde, essayant d'accorder signe et objet. Exemple de cette volonté de faire concorder la graphie du nom et l'objet qu'il évoque dans Notes prises pour un oiseau: «Le mot OISEAU : il contient toutes les voyelles. Très bien, j'approuve. Mais, à la place de l's, comme seule consonne, j'aurais préféré l'L de l'aile : oileau, ou le $\mathrm{V}$ du bréchet, le $\mathrm{V}$ des ailes déployées, le $\mathrm{V}$ d'avis : oiveau ». Le vieux moissonneur, jubilant à la lecture-écriture du monde, peut se réjouir : il peut au moins s'envoler à l'aide du mot allemand : celui-ci commence avec un $\mathrm{V}$... et finit avec un $\mathrm{L}$ !

21 Mais, comme dans tout conte, le monde enchanteur est éphémère. Une pluie diluvienne s'abat sur le plateau, et la vue sur celui-ci disparaît, donc tout signe. Ce "déluge » (Sinflut) ressemble étrangement à une malédiction. On n'échappe pas aussi facilement au monde moderne, et cette catastrophe est comme le signe avant-coureur du prochain retour à la ville. J'ai déjà parlé de cette scène dans la grotte lors de laquelle la femme dénigre les soi-disant s pouvoirs du vieil homme, lui lançant notamment : «Du bist ein schlechter Léser», et ironisant sur le fait que les trois lettres «dim» n'avaient rien à voir 
avec Mithra mais qu'il s'agissait d'une marque de collant! Où est le vrai ? N'était-ce qu'un songe, ou une illusion, cette moisson sur le haut-pays ? Là apparaît au grand jour le débat, ou plutôt le combat qui est au centre du conte : celui entre la femme-sorcière et le vieillard-magicien (ou prétendant l'être). À l'idéal d'union avec le réel au moyen du langage qu'a énoncé le vieil homme s'oppose violemment le désespoir, voire la méchanceté (que nous découvrirons finalement superficielle, plus un symptôme qu'un trait de caractère) de la femme. Si le vieil homme a le désir d'un rapport fécond avec les choses, certain que hors des villes la beauté est encore accessible, la femme quant à elle affirme sa répugnance pour tout ce qui l'entoure - mis à part tout de même un possible amant -, parce que pour elle tout, en ville ou en dehors, est laid et sans intérêt. Elle ressemble à quantité de gens qui, dégoûté par la vie dans les grandes métropoles (et parfois sans s'en rendre compte), finissent par trouver insupportable aussi tout le reste. Lors de son attaque contre son compagnon, la femme prononce cette parole sans appel : «Auch hierzuland ist leer leer geworden, tôt tôt, das Vergangene unwiderruflich, und zu uberliefern ist nichts mehr». Conditionnée par le monde urbain elle a perdu jusqu'à la liberté de se donner un autre cadre de pensée et de vie. N'ayant plus de regard pour le monde naturel, elle le met sur le même plan que cet univers citadin qui l'aliène, faute d'avoir le désir indispensable de trouver un regard neuf.

Quelle est la cause de cette crise soudaine chez la femme, après qu'elle a tout de même pu se faire une idée de « l'autre vision » que recherche son compagnon? N'apparaît-elle pas, cette crise, à ce moment du conte, comme injustifiée, et simple volonté de dramatisation de la part de Handke? Bien au contraire, elle semble découler de cette expérience du haut-pays, "pays ambigu ", dit la voix narrative. Quittés la ville et son grouillement d'images il faut en effet faire l'expérience du désert et de la solitude.

Le paysage du haut-pays apparaît nu, décharné - un squelette. Les arbres, écrit Handke, sont "desséchés, sans écorce, des troncs cendreux qui émergent de l'herbe pâle». Regardons les tableaux de Cézanne : les verts ne sont jamais vifs, domine le gris de la rocaille et le bleu-gris du ciel. Voilà le paysage que découvrent les compagnons du vieil homme et qui enchante ce dernier (ainsi que le soldat) : y règne le vent. La contrée paraît "tout à fait morte", et "même les arbres sains prennent une silhouette de ruines ». Comment dès lors vivre là ? L'adaptation à cet espace ne peut être qu'une ascèse, surhumaine pour les anciens citadins. Cela explique la panique de la femme au moment de sa découverte du lieu. Le haut-pays fait peur, là, « c'est l'hiver en été » dit le vieil homme. Dès le voyage en train il était pourtant clair que le lieu recherché serait un lieu abandonné.

C'est à cause de cette désertion des lieux jadis habités que la mort semble rôder dans ces paysages du conte. Le plus désert, le plus inquiétant donc de ces paysages, c'est ce «no man's land», vide de toute image artificielle, sociale servant à contrôler les esprits, lieu de la disparition du «magicien». Quantité de détails y évoquent la désolation, détails souvent négatifs. C'est d'abord un "désert de sable, au milieu du haut-pays ». On ne trouve là que des plantes mortes, l'arbre au centre du désert parait lui aussi mort, et "même la petite piste de fourmis (est) dépeuplée et les trous dans le sol (sont) vides". Ces quelques détails déstabilisent le lecteur; ce dernier peut se demander en effet si ces signes morbides ne prouvent pas l'échec du disparu, et à bon droit croire avec la femme que ce « royaume du monde » tant célébré par le vieil homme est une utopie. C'est là qu'il nous faut comprendre ce qu'est vraiment le " pays ambigu ", ce à quoi peut nous aider la citation d'un texte chinois, Le vrai livre du pays du sud en fleurs, placée à la fin du 
conte par Handke : «La vie de l'homme sur terre est vite passée comme le reflet d'un cheval blanc, à travers une fente... Essaie de faire route avec moi jusqu'au château du non-être, où tout est un ». Cette dernière phrase, rappelant celle de Goethe que j'ai citée au début de cet article, cette dernière phrase, même si elle reste hermétique, éclaire tout de même toute une série d'éléments contradictoires du conte, dont celui que je viens d'évoquer (signes de vie et d'un possible ressourcement dévoilés par le vieillard s'opposant aux signes de mort peuplant les paysages révélés), et même la nature profondément contradictoire du conte, du moins pour un esprit occidental. Il ne faut pas voir en effet ce "pays ambigu» comme un pays tragique exprimant la déchéance de la nature, mais plutôt comme l'espace du grand dépouillement, où tout ce qui n'est pas essentiel meurt, et où doivent se rejoindre conscience de la vie et conscience de la mort parce qu'elles ne sont pas, fondamentalement, séparables, comme pour ces penseurs taoïstes chinois tels Tchouang-Tseu qui écrit que «la distinction de la vie et de la mort n'est pas réelle », qu'une conscience peut et doit naître dépassant cette contradiction et élaborer une sensation du monde où tous les « contraires » fusionnent. Le haut-pays est lieu de vie et de mort en même temps parce que, par exemple, le vent y court sans arrêt, vent passager, « mortel », comme l'est tout ce qui vit sur le plateau - ne s'y déroulent que des «transmutations » et non des morts. Handke situe dans le conte cet espace où doivent nécessairement être dépassées toutes les "contradictions", qui ne sont que vues de l'esprit, et rejointe une unité. "Le château du non-être où tout est un ", c'est la conscience apaisée à laquelle doivent accéder les héros du conte - conscience qui n'oppose plus, aussi, parole et silence. «Allein in die Stille/ Allein die Stille» chante le vieil homme dans son désert, et ce silence permet, s'il est trouvé, l'invention d'une "écriture de silence ", écriture que les quatre personnages, plus ou moins consciemment, désirent réaliser, parce que cette écriture est dégagement, ouverture sur un horizon, et que réalisèrent il y a très longtemps des poètes chinois comme Li Po, Wang Wei ou Han Shan. Bien sûr, ces personnages auraient pu tenter de trouver cette écriture en ville. Mais ils auraient dû faire comme ce père du soldat qui ne peignait que «les espaces intermédiaires étroits comme des rainures » entre les maisons, d'où la difficulté pour lui d'accéder à une conscience dégagée, gêné qu'il était par chaque rue le cernant ; le fils, lui, quitte la ville pour aller jusqu'au bout dans cette quête qu'avait commencée, à sa façon, son père.

De plus, c'est dans cet espace $d u$ «Hochland» qu'une convivialité peut réellement exister entre les êtres. Errant sur le plateau, les personnages ne sont pas séparés les uns des autres, ils sont unis par la même recherche, et se font de temps à autre des signes de la main. Cette convivialité est essentiellement silencieuse, laissant place quelquefois à des monologues de chacun des personnages qui renforcent l'amitié qu'ils ressentent, au fond, les uns pour les autres, même si cela ne va pas sans heurt (comme entre le vieil homme et la femme). Sans doute est-ce grâce à cette amitié découverte auprès du vieil homme qu'une fois celui-ci disparu, les autres n'arrivent pas à croire à sa disparition; il ne nous a, pensent-ils simplement, que devancés, et il nous attend plus loin... Le vieillard leur est devenu indispensable, et ils pensent pouvoir le rejoindre grâce à une carte que celui-ci a laissée. Cette carte les mène jusqu'à une ville inconnue, sombre, où vient d'avoir lieu un tremblement de terre. C'est seulement une fois arrivés dans cette ville mille fois plus étouffante que celle du départ qu'ils se rendent compte du fait - et la femme aussi - que l'«Entzifferer» n'était pas un «vieux fou», et que sa recherche du «Hochland» était non seulement justifiée mais bénéfique. 
Dans un entretien, André Dhôtel dit que dans toute bonne histoire, « la difficulté, c'est qu'il y ait un arrêt, que le lecteur, à un moment donné, s'arrête et voit quelque chose ». Alors doit avoir lieu une discussion, les personnages de l'histoire doivent reprendre un à un les détails et les événements de l'aventure. De cette manière, dit Dhôtel, se produit une "entrée en conversation " entre personnages et auteur, personnages et lecteur, auteur et lecteur. C'est exactement ce qui se passe dans cette scène finale de Die Abwesenheit. D'abord le silence s'installe entre la femme, le soldat et le joueur. Chacun retrouve sa solitude malheureuse, et croit reconnaître par instants, dans l'hôtel où ils logent tous les trois ensemble, le vieil homme ; mais ce n'est jamais lui. C'est le temps de "l'absence maléfique ", comme si l'absent avait fait exprès de les abandonner pour que ceux-ci se laissent aller au désespoir, au sentiment d'avoir fait une perte irréparable, puis - et là nous pouvons dire que de maléfique l'absence devient bénéfique parce qu'elle les force à remédier à l'absence -, ils partent à la recherche de la présence (du vieil homme et du monde, tous deux confondus). Mais avant ce départ a lieu ce que Dhôtel appelle la discussion, lors de laquelle apparaissent tous les problèmes posés par le conte. Il est en effet très possible, à la lecture du livre, de croire avec la femme que le vieil homme est un illuminé ou un charlatan. Dans une scène du début du livre, ne le voyons-nous pas suivre un "groupe de débiles", reconnaissant "les siens »? Et juste avant, à son départ de l'hospice, lorsque le personnel et le directeur le saluent avec courtoisie, n'est-il pas suggéré qu'ils ne font là que jouer la comédie, et prennent leur pensionnaire ni plus ni moins que pour un grabataire aux habitudes extravagantes? Cette dernière partie laisse donc le champ libre à une interprétation personnelle du "vieil original», comme elle laisse le choix aux trois autres personnages de partir ou non à la recherche de leur compagnon. Le soldat lui-même hésite, et les deux autres avec lui :

Mag sein: Mein Entschwundener war eine Art Heiratsschwindler mit der Frau Welt. Er hat einen Bund versprochen und ihn nicht gehalten. [...] Er war ein falscher Fürst und hat mich weg von zuhause, weg von der Kaserne, weg von meinesgleichen in ein Land gelockt, wo es ausser vielleicht für ihn keine Lebenluft bigt. Kein Fürst eines gemeinsamen Weltreichs war er, sondern dessen Gaukler.

Le soldat, comme la femme, est assez dur avec le vieil homme. En vérité, il aurait aimé que celui-ci les emmène jusqu'à une terre paradisiaque et éternellement belle, et que n'ait pas eu lieu cette tempête les chassant tous les quatre de leur domaine. Seulement, la leçon et l'idéal du vieillard sont autres : selon lui le découvreur ne doit pas s'installer dans une vision du réel, mais a pour tâche au contraire de ne jamais cesser de changer sa vision, d'aller toujours plus avant dans son exploration du monde, monde qui demeure toujours inconnu. Ce n'est qu'ensuite que le soldat raconte son rêve où le Disparu réapparaît sous son vrai visage. Il y est dit de lui : « Le vieil homme n'était pas mal intentionné, il était l'éternel enfant. Il ne faut pas que la matière de l'enfance s'use ». Alors, laquelle croire de ces deux voix ? À chacun de choisir, nous dit Peter Handke à la fin du conte, entre l'appel du vieillard-enfant et le malheur initial de ses trois compagnons, à chacun de choisir entre une conscience en quête d'un monde beau quoiqu'incertain, ressemblant fort au «monde flottant " de l'imaginaire japonais, et une conscience ordinaire, insatisfaite, mais ayant du moins l'avantage d'être figée dans son rapport à un univers invariable et commun à tous. 


\section{RÉSUMÉS}

Peter Handke poursuit dans Die Abwesenheit la quête d'un "merveilleux » déjà commencée dans Die Lehre der Sainte Victoire, livre dans lequel l'écrivain raconte sa découverte de la peinture de Cézanne et du lieu de révélation capital : la montagne, le haut pays. Les personnages du conte de Handke partent comme l'écrivain dans le texte autobiographique de 1980 à la recherche d'une autre sensation, sensation merveilleuse car elle révèle les arcanes du monde naturel et artistique. Les personnages ont certes quelques ressemblances avec des héros légendaires de certains récits traditionnels - roi, sorcière ou magicien -, mais en eux sont aussi reconnaissables les quatre éléments nécessaires au Grand (đuvre alchimique : l'Eau (le vieil homme), la Terre (le joueur), l'Air (le soldat), et le Feu (la femme). Ce symbolisme alchimique nous mène à une autre dimension du livre. Chez Handke, le conte n'est plus sortie du réel et accès à un monde imaginaire, mais évasion d'un réel urbain aliénant et entrée dans le "Réel des réels " (Die Lehre der Sainte Victoire), ou, autre expression de l'auteur, dans le « Royaume du monde ». Le réel se dévoile espace merveilleux, où chaque objet est magique et où le voir veut aussi dire l'exprimer (n'oublions pas que Handke a traduit Francis Ponge, grand poète des choses). Il faut donc parler de cet autre Espace-temps du conte, et voir à quels actes celui-ci conduit les personnages pour qu'ils en deviennent les initiés. Là intervient la nécessité de ce que j'appelle une «lecture du monde ", espèce de moisson de sensations et de mots que feront les héros arrivés au «Hochland». Mais peut-on demeurer dans cet espace différent ? Dans tout conte le merveilleux est éphémère. L'« oasis du vide », comme l'écrit Handke, est vite ravagé par le monde moderne. L'espace du conte devient chez l'écrivain autrichien ce lieu ambigu où se confrontent un réel magique fragile et un univers moderne désenchanté envahissant peu à peu tout ce qui n'y participe pas.

Peter Handke nimmt in Die Abwesenheit die Suche nach dem «Wunderbaren» wieder auf, welche er bereits in Die Lehre der Sainte Victoire begonnen hat, einem Buch in dem der Autor seine Entdeckung der Malerei von Cezanne und dem Gebirge, dem Hochland, als einem wesentlichen Ort der Offenbarung erzählt. Die Figuren des Märchens von Handke brechen auf wie der Autor des autobiographischen Textes von 1980 auf der Suche nach einer wunderbaren Empfindung, wunderbar, weil sie die Arkanen der natürlichen und der künstlerichen Welt offenbart. Die Figuren haben sicherlich einige Ähnlichkeiten mit legendären Helden traditioneller Berichte König, Hexe oder Zauberer - aber man kann in ihnen auch die vier zum alchemischen Werk benötigten Elemente wiedererkennen: das Wasser (der Alte), die Erde (der Spieler), die Luft (der Soldat) und das Feuer (die Frau). Diese alchemische Symbolismus führt uns zu einer weiteren Dimension des Buchs. Bei Handke ist das Märchen kein Ausgang aus der Realität und Eintritt in eine imaginäre Welt sondern der Ausbruch aus der städtischen anfeindeden Realität und Zugang zu dem «Allerwirklichsten» (Die Lehre der Sainte Victoire), oder dem «reich der Welt», ein anderer Ausdruck des Autors. Das Wirkliche enthüllt sich als wunderbarer Raum, in dem jeder Gegenstand magisch ist, und dessen Wahrnehmung auch dessen Ausdruck bedeutet (vergessen wir nicht, dass Handke Francis Ponge, grosser Dichter der Gegenstände, übersetzte). Die Figuren agieren also im rahmen eines anderen Raums und einer anderen Zeit, in welchen bzw welche sie eingeweiht werden. Daher die Notwendigkeit einer Lektüre der Welt, wie ich es nenne, eine Art Ernte der Eindrücke und Wörter, die die Helden im Hochland angekommen machen werden. Aber kann man in diesem anderen Raum bleiben? In jedem Märchen ist das Wunderbare vergänglich. «Die Oase der Leere», wie Handke schreibt, ist schnell zerstört durch die Moderne. Der Raum der Erjählung wird bei dem österreichischen Schriftsteller dieser ambivalente Ort, in dem magische zerbrechliche Wirklichkeit und die banale Moderne aufeinanderstossen, welche peu ä peu alles erfasst. 